\title{
Systematic Review \\ Pharmacokinetic Outcomes of the Interactions of Antiretroviral Agents with Food and Supplements: A Systematic Review and Meta-Analysis
}

\author{
Tippawan Siritientong ${ }^{1,2, *(\mathbb{D})}$, Daylia Thet ${ }^{1}\left(\mathbb{D}\right.$, Janthima Methaneethorn ${ }^{3,4}\left(\mathbb{D}\right.$ ) and Nattawut Leelakanok ${ }^{5}(\mathbb{D}$ \\ 1 Department of Food and Pharmaceutical Chemistry, Faculty of Pharmaceutical Sciences, Chulalongkorn \\ University, Bangkok 10330, Thailand; daliathet@gmail.com \\ 2 Center of Excellence in Burn and Wound Care, Chulalongkorn University, Bangkok 10330, Thailand \\ 3 Pharmacokinetic Research Unit, Department of Pharmacy Practice, Faculty of Pharmaceutical Sciences, \\ Naresuan University, Phitsanulok 65000, Thailand; janthima.methaneethorn@gmail.com \\ 4 Center of Excellence for Environmental Health and Toxicology, Naresuan University, \\ Phitsanulok 65000, Thailand \\ 5 Department of Clinical Pharmacy, Faculty of Pharmaceutical Sciences, Burapha University, \\ Chonburi 20131, Thailand; nattawut.le@go.buu.ac.th \\ * Correspondence: tippawan.s@pharm.chula.ac.th; Tel.: +66-61-956-2898
}

check for updates

Citation: Siritientong, T.; Thet, D.; Methaneethorn, J.; Leelakanok, N. Pharmacokinetic Outcomes of the Interactions of Antiretroviral Agents with Food and Supplements: A

Systematic Review and

Meta-Analysis. Nutrients 2022, 14, 520. https://doi.org/10.3390/ nu14030520

Academic Editor: William B. Grant

Received: 16 December 2021

Accepted: 23 January 2022

Published: 25 January 2022

Publisher's Note: MDPI stays neutral with regard to jurisdictional claims in published maps and institutional affiliations.

Copyright: (c) 2022 by the authors. Licensee MDPI, Basel, Switzerland. This article is an open access article distributed under the terms and conditions of the Creative Commons Attribution (CC BY) license (https:// creativecommons.org/licenses/by/ $4.0 /)$.

\begin{abstract}
Because pharmacokinetic changes in antiretroviral drugs (ARV), due to their concurrent administration with food or nutritional products, have become a clinical challenge, it is necessary to monitor the therapeutic efficacy of ARV in people living with the human immunodeficiency virus (PLWH). A systematic review and meta-analysis were conducted to clarify the pharmacokinetic outcomes of the interaction between supplements such as food, dietary supplements, and nutrients, and ARV. Twenty-four articles in both healthy subjects and PLWH were included in the qualitative analysis, of which five studies were included in the meta-analysis. Food-drug coadministration significantly increased the time to reach maximum concentration $\left(t_{\max }\right)(p<0.00001)$ of ARV including abacavir, amprenavir, darunavir, emtricitabine, lamivudine, zidovudine, ritonavir, and tenofovir alafenamide. In addition, the increased maximum plasma concentration $\left(C_{\max }\right)$ of $A R V$, such as darunavir, under fed conditions was observed. Area under the curve and terminal half-life were not significantly affected. Evaluating the pharmacokinetic aspects, it is vital to clinically investigate ARV and particular supplement interaction in PLWH. Educating patients about any potential interactions would be one of the effective recommendations during this HIV epidemic.
\end{abstract}

Keywords: food-drug interactions; nutrients; pharmacokinetics; HIV; AIDS

\section{Introduction}

The human immunodeficiency virus (HIV) infection is one of the major health challenges around the world. Approximately 33 million people have died from HIV/acquired immunodeficiency syndrome (AIDS)-related illness since the start of the HIV epidemic worldwide [1]. Globally, over 37 million people were living with HIV (PLWH) in 2020, of which more than 27 million were receiving HIV treatment or antiretroviral (ARV) therapy [2]. The commonly adopted ARV therapy includes the combined use of three or more ARVs from at least two different classes, such as nucleoside reverse transcriptase inhibitor (NRTI), non-nucleoside reverse transcriptase inhibitor (NNRTI), and protease inhibitor (PI). In adults and adolescents, tenofovir/lamivudine or tenofovir/emtricitabine is the preferred backbone, which is used in combination with a third drug, dolutegravir for post-exposure prophylaxis [3]. Due to the widespread use of effective ARV therapy and HIV care, about $66 \%$ of the overall infected population achieve viral suppression [2].

Dietary supplements including vitamins and minerals are widely used to boost the body's defense system in many patients with nutrient deficiencies. European Food Safety 
Authority stated food supplements as sources of nutrients such as vitamins and minerals, which have nutritional or physiological value for the regulation of nutritional deficiencies [4]. In PLWH, inadequate nutrient intake becomes a clinical concern that could potentiate treatment failure. A previous report found that PLWH did not achieve the dietary recommendations of energy and micronutrients, especially zinc and iron [5,6]. To meet the required level of protein and other nutrients, PLWH use a variety of supplements in addition to their normal daily treatment therapy for HIV $[7,8]$. The use of complementary medications may be quite popular since the products are easily accessible over the counter. The lifetime use of complementary and alternative medicine was 30-90\% prevalent in PLWH, commonly using vitamins, minerals, and other over-the-counter supplements [9].

In susceptible patients, even substantially small effects of food-drug interaction may result in therapeutic changes for some drugs with a narrow therapeutic index [10]. Likewise, food-drug interaction may influence the therapeutic efficacy of the drug by changing pharmacokinetic processes such as absorption, distribution, metabolism, and excretion, or pharmacodynamic physiological effects of the drug [11]. Given the different mechanisms of interactions between ARV and nutrients, it is critical to monitor the resulting implications that may positively or negatively affect the therapeutic outcomes. Due to the effect on pharmacokinetic parameters such as maximum plasma concentration $\left(C_{\max }\right)$, area under the curve (AUC), time to reach maximum concentration $\left(t_{\max }\right)$, and terminal half-life $\left(t_{1 / 2}\right)$, food considerations are essential in the treatment of HIV. The effect of food on the absorption of ARV was well noted following the reduction in plasma concentration of indinavir [12]. Food containing high-fat contents is likely to reduce the rate and extent of the absorption of oral drugs by delaying gastric emptying, whereas some meals with high-protein contents would increase the extent of oral drug absorption by stimulating intestinal transporters and enzyme activity [13]. However, the effects of food on particular oral drugs may not always be of clinical importance as some interactions may not occur in all patients. Although many ARVs can be taken with food to optimize their absorption, the concurrent administration with food might result in a decreased rate of absorption, longer $t_{\max }$, and declined $C_{\max }$ of some ARV such as zidovudine and lamivudine; although, they were not clinically significant [14]. Likewise, the subtherapeutic levels of raltegravir due to concomitant use of calcium supplement in an HIV-infected patient were reported [15]. Unpredictable and variable drug concentrations are major problems that lead to treatment failure or adverse reactions. The possible interaction of concurrent food and ARV has become a clinical challenge in PLWH. This systematic review and meta-analysis aims to investigate the effect of food, dietary supplements, or nutrients on pharmacokinetic outcomes of ARV by comparing the pharmacokinetic parameters in either PLWH or healthy people with and without supplements. The important role of food in the ARV era is not much known in clinical settings. The extent of changes in plasma concentration-time profiles of ARV during fed and fasted conditions can evaluate the potential interaction.

\section{Materials and Methods}

\subsection{Literature Search}

According to the Preferred Reporting Items for Systematic Reviews and Meta-Analyses (PRISMA) guidelines, a systematic literature search was conducted [16]. Four databases including Cochrane Library, ScienceDirect, Scopus, and PubMed were searched from the date of their inception to June 2021 to identify studies that explored supplements and ARV interactions. The search terms such as (food supplement OR dietary supplement OR nutrient supplement) AND (antiretroviral therapy OR antiretroviral drug OR nonnucleoside reverse transcriptase inhibitor OR nucleoside reverse transcriptase inhibitor OR protease inhibitor) AND (area under curve OR cytochrome P450 OR plasma concentration) were used. No filters or restrictions were applied during the search. The full search strategies are mentioned in Supplementary Material Table S1. The full protocol of this systematic review and meta-analysis was not registered. 


\subsection{Selection Criteria}

The primary outcomes that we aimed to collect were AUC, $C_{\max }, t_{\max }$, and $t_{1 / 2}$ which are parameters used for primarily assessed the food effect on drug pharmacokinetics [17]. All articles of any language, year published, and country that reported nutrient or food supplement and ARV interactions were included to retrieve relevant studies. Only studies that reported pharmacokinetic outcomes such as AUC, $C_{\max }, t_{\max }$, and $t_{1 / 2}$ were included in the meta-analysis. Review articles, book chapters, conference abstracts, posters, in vitro studies, and animal studies were excluded. The search results were exported to a citation manager (Endnote 20.1., Clarivate Analytics, New York, NY, USA). Titles and abstracts were thoroughly screened, and eligible studies were independently selected by the first two authors for inclusion in this systematic review. Specific characteristics for inclusion were studies of adult healthy people or PLWH on ARV, which discussed changes in ARV levels, concerned adverse events, or treatment failure directly resulting from the food-drug interaction (Phase I-IV clinical trials). Disagreements between the first two authors were thoroughly resolved by consensus, and further by discussing among four authors for final inclusion. The PRISMA diagram for the complete literature search is shown in Figure 1.

\subsection{Data Extraction}

After full-text articles were screened for inclusion in the systematic review, data extraction was performed. Information such as author name, year of publication, year of study, study design, study setting, characteristics of participants, types of ARV, types of supplements, and pharmacokinetic outcomes were extracted by the first author and the data entry was judged by co-authors. Discrepancies were discussed among all authors. Any disagreement was resolved by consensus. 


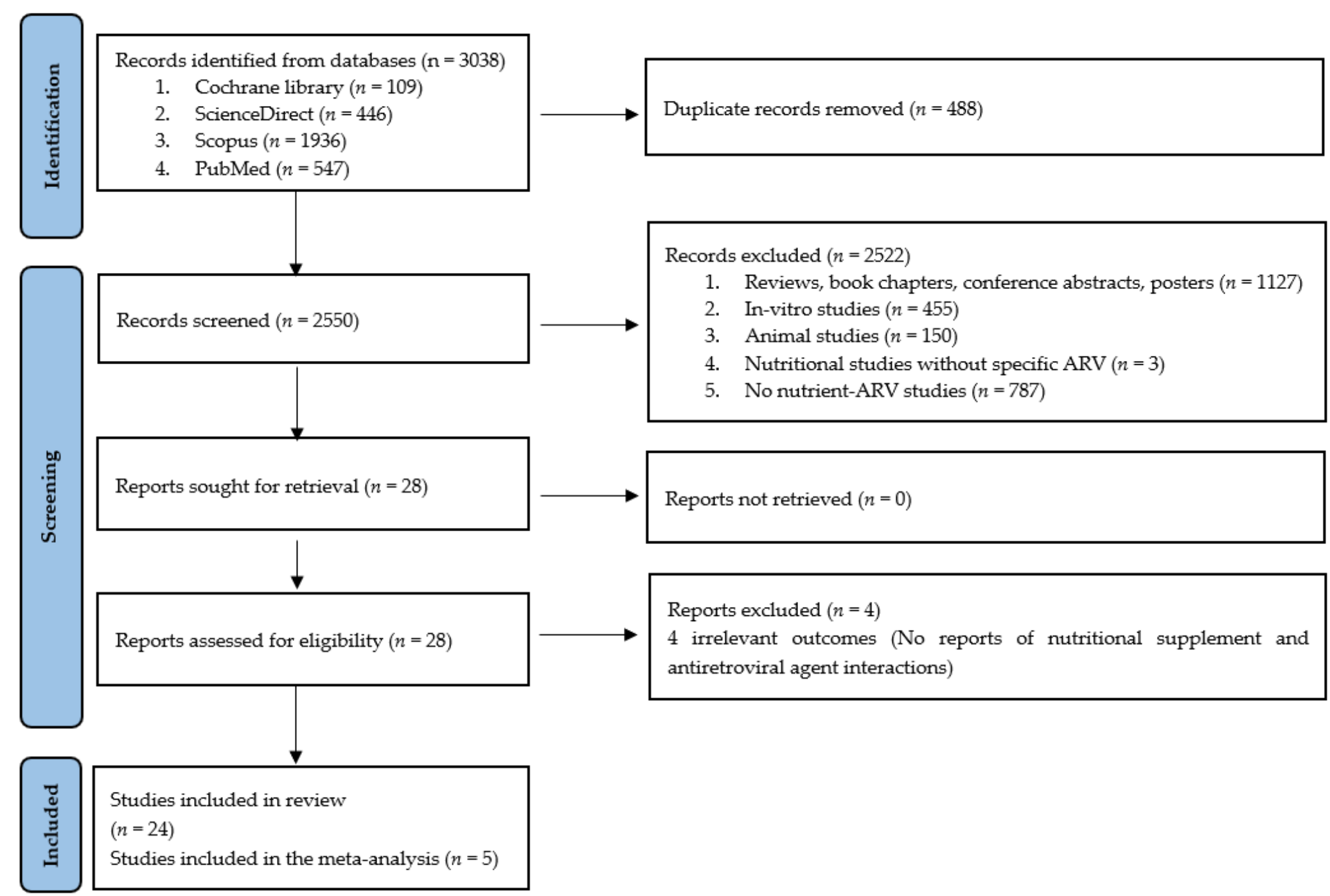

Figure 1. Flow diagram for selection and inclusion of the studies. 


\subsection{Quality Assessment}

The risk of bias of the included studies was assessed using the different validated tools including the risk of bias assessment tools for primary and secondary medical studies [18], ROBINS-I for non-randomized studies [19], Cochrane Collaboration's tool for randomized trials [20], a modified Newcastle-Ottawa Quality Assessment Scale for cross-sectional studies [21], a modified tool for case report described by Murad et al. [22], and Cochrane risk of bias for crossover studies [23]. The quality assessment of included studies is mentioned in Supplementary Material Table S2.

\subsection{Statistical analysis}

Review Manager (RevMan version 5.4.1: The Nordic Cochrane Center, Copenhagen, Denmark) was used for conducting meta-analysis. Random effect models with the inverse variance method were used to calculate the weights of the studies. Publication bias was assessed by visually inspecting the funnel plot. The heterogeneity among studies was assessed by $\mathrm{I}^{2}$ statistics. The $\mathrm{I}^{2}$ of $<25 \%$ was assumed as negligible heterogeneity, whereas that of $>75 \%$ was high heterogeneity [20].

\section{Results}

A total of 3038 articles were retrieved. After duplicates and irrelevant studies were removed by reviewing titles and abstracts, 28 articles were selected for potential inclusion. The number of records with reasons for exclusion is summarized in Figure 1. Records that possibly met the inclusion criteria were assessed for eligibility for systematic review. Finally, twenty-four articles were included in the systematic review. Thirteen articles of them were good quality and eleven articles were fair (Supplementary Material Table S2). In general, the heterogeneity was high, and the $95 \%$ confidence intervals of the pooled data were close to the most weighted and included studies whose intervals were narrow. The summary description of the included studies is characterized in Table 1. 
Table 1. Description of the included studies.

\begin{tabular}{|c|c|c|c|c|c|c|}
\hline $\begin{array}{l}\text { Author, Year of } \\
\text { Publication }\end{array}$ & Study Setting & Study Design & Study Participants & Food or Dietary Supplements & $\begin{array}{c}\text { Antiretroviral } \\
\text { Drugs }\end{array}$ & Major Outcomes \\
\hline $\begin{array}{l}\text { Kupferschmidt } \\
\text { et al., } 1998 \text { [24] }\end{array}$ & Switzerland & $\begin{array}{l}\text { Open crossover } \\
\text { study }\end{array}$ & $\begin{array}{l}8 \text { healthy male } \\
\text { subjects, mean age } \\
26 \pm 2 \text { years }\end{array}$ & $\begin{array}{l}400 \mathrm{~mL} \text { grapefruit juice (Hitchcock, } \\
\text { freshly prepared), taken } 45 \text { min and } \\
15 \text { min before intravenous or } \\
\text { oral saquinavir }\end{array}$ & $\begin{array}{l}12 \text { mg intravenous } \\
\text { saquinavir or } 600 \\
\text { mg oral saquinavir }\end{array}$ & $\begin{array}{l}\text { - Grapefruit juice enhances the oral } \\
\text { bioavailability of saquinavir without } \\
\text { affecting its clearance. } \\
\text { - } \quad \text { AUC of saquinavir increased after } \\
\text { pretreatment with grapefruit juice. }\end{array}$ \\
\hline $\begin{array}{c}\text { Moore et al., } \\
1999 \text { [14] }\end{array}$ & $\begin{array}{l}\text { United States } \\
\text { of America }\end{array}$ & $\begin{array}{l}\text { Single-center, } \\
\text { open-label, } \\
\text { randomized, } \\
\text { three-way } \\
\text { crossover study }\end{array}$ & $\begin{array}{l}24 \text { healthy } \\
\text { subjects, mean age } \\
26 \pm 4.4 \text { years }\end{array}$ & $\begin{array}{l}\text { The standardized high-fat breakfast: } \\
2 \text { slices of toasted white bread with } \\
\text { butter, } 2 \text { eggs fried in butter, } 2 \text { slices } \\
\text { of bacon, } 2 \text { ounces of hash-browned } \\
\text { potatoes, and } 8 \text { ounces of } \\
\text { whole milk }\end{array}$ & $\begin{array}{l}150 \text { mg lamivudine, } \\
300 \mathrm{mg} \text { zidovudine, } \\
\text { taken within } 5 \mathrm{~min} \\
\text { after breakfast }\end{array}$ & $\begin{array}{l}\text { - The extent of absorption of } \\
\text { lamivudine and zidovudine from the } \\
\text { combination tablet was not altered by } \\
\text { administration with meals. } \\
\text { - The absorption rate }\left(\mathrm{t}_{\max } \text { and } \mathrm{C}_{\max }\right) \\
\text { of lamivudine and zidovudine } \\
\text { was reduced. } \\
\text { Since these changes were not } \\
\text { considered clinically important, the } \\
\text { drug formulation can be taken with } \\
\text { or without food. }\end{array}$ \\
\hline $\begin{array}{l}\text { Yuen et al., } \\
2001 \text { [25] }\end{array}$ & $\begin{array}{l}\text { United States } \\
\text { of America }\end{array}$ & $\begin{array}{l}\text { Single-center, } \\
\text { open-label, } \\
\text { randomized, } \\
\text { three-way } \\
\text { crossover study }\end{array}$ & $\begin{array}{l}24 \text { healthy } \\
\text { subjects, mean age } \\
37.6 \pm 9.6 \text { years }\end{array}$ & $\begin{array}{l}\text { The standardized high-fat breakfast: } \\
2 \text { slices of toasted white bread with } \\
\text { butter, } 2 \text { eggs fried in butter, } 2 \text { slices } \\
\text { of bacon, } 2 \text { ounces of hash-browned } \\
\text { potatoes, and } 8 \text { ounces of } \\
\text { whole milk }\end{array}$ & $\begin{array}{l}300 \mathrm{mg} \text { abacavir, } \\
150 \mathrm{mg} \text { lamivudine, } \\
300 \mathrm{mg} \text { zidovudine, } \\
\text { taken within } 5 \text { min } \\
\text { after breakfast }\end{array}$ & $\begin{array}{l}\text { The extent of absorption of abacavir, } \\
\text { lamivudine, and zidovudine from the } \\
\text { combination tablet was not altered by } \\
\text { administration with meals. } \\
\text { The absorption rate }\left(\mathrm{t}_{\max } \text { and } \mathrm{C}_{\max }\right) \\
\text { of abacavir, lamivudine, and } \\
\text { zidovudine were reduced. } \\
\text { - Since these changes were not } \\
\text { considered clinically important, the } \\
\text { drug formulation may be taken with } \\
\text { or without food. }\end{array}$ \\
\hline
\end{tabular}


Table 1. Cont.

\begin{tabular}{|c|c|c|c|c|c|c|}
\hline $\begin{array}{l}\text { Author, Year of } \\
\text { Publication }\end{array}$ & Study Setting & Study Design & Study Participants & Food or Dietary Supplements & $\begin{array}{c}\text { Antiretroviral } \\
\text { Drugs }\end{array}$ & Major Outcomes \\
\hline $\begin{array}{l}\text { Penzak et al., } \\
2002 \text { [26] }\end{array}$ & $\begin{array}{l}\text { United States } \\
\text { of America }\end{array}$ & $\begin{array}{l}\text { Open-label, } \\
\text { randomized, } \\
\text { crossover study }\end{array}$ & $\begin{array}{l}13 \text { healthy } \\
\text { subjects, mean age } \\
24 \pm 1.9 \text { years }\end{array}$ & $\begin{array}{l}8 \text { ounces of Seville }{ }^{\circledR} \text { orange juice } \\
\text { (prepared by squeezing fresh fruit) } \\
\text { or grapefruit juice (prepared from } \\
\text { frozen concentrate), taken together } \\
\text { with indinavir }\end{array}$ & $800 \mathrm{mg}$ indinavir & $\begin{array}{l}\text { - } \text { Coadministration of Seville }^{\circledR} \text { orange } \\
\text { juice and indinavir resulted in a } \\
\text { statistically significant increase in } \\
\text { indinavir } t_{\text {max without altering other }} \\
\text { pharmacokinetic parameters. } \\
\text { Coadministration of grapefruit juice } \\
\text { and indinavir did not significantly } \\
\text { change indinavir pharmacokinetic } \\
\text { parameters including } A_{U} C_{0-5} \\
\text { AUC }_{0-8}, C_{\min }, C_{\max }, \mathrm{t}_{1 / 2}, \text { and oral } \\
\text { clearance of indinavir. }\end{array}$ \\
\hline \multirow[b]{2}{*}{$\begin{array}{l}\text { Falcoz et al., } \\
2002 \text { [27] }\end{array}$} & \multirow[b]{2}{*}{ Germany } & $\begin{array}{l}\text { Study A: } \\
\text { Single-center, } \\
\text { open-label, } \\
\text { single-dose, } \\
\text { randomized, } \\
\text { five-way } \\
\text { crossover study }\end{array}$ & $\begin{array}{l}16 \text { healthy male } \\
\text { subjects, age range } \\
24-50 \text { years }\end{array}$ & $\begin{array}{l}\text { The standardized high-fat breakfast: } \\
2 \text { slices of toasted white bread with } \\
\text { butter, } 2 \text { eggs fried in butter, } 2 \text { slices } \\
\text { of bacon, } 2 \text { ounces of hash-browned } \\
\text { potatoes, and a glass of whole milk }\end{array}$ & $\begin{array}{l}1656 \mathrm{mg} \\
\text { GW433908A, } 1728 \\
\text { mg GW433908G } \\
\text { (taken within } 5 \text { min } \\
\text { after breakfast), } \\
2592 \mathrm{mg} \\
\text { GW433908G, } \\
1200 \mathrm{mg} \\
\text { amprenavir }\end{array}$ & \multirow{2}{*}{$\begin{array}{l}\text { The effect of food on GW433908G } \\
\text { pharmacokinetic parameters } \\
\text { (AUC } \mathrm{A}_{0-\infty}, \mathrm{C}_{\max }, \mathrm{t}_{\max } \text {, and oral } \\
\text { bioavailability) was not statistically } \\
\text { significant. } \\
\text { The therapeutic levels of amprenavir } \\
\text { prodrug under fed conditions and } \\
\text { fasting state were comparable. }\end{array}$} \\
\hline & & $\begin{array}{l}\text { Study B: } \\
\text { Open-label, } \\
\text { single-dose, } \\
\text { randomized, } \\
\text { six-way crossover } \\
\text { bioequivalence } \\
\text { study }\end{array}$ & $\begin{array}{l}24 \text { healthy male } \\
\text { subjects, age range } \\
19-48 \text { years }\end{array}$ & $\begin{array}{l}\text { The standardized high-fat } \\
\text { breakfast: } 2 \text { slices of toasted } \\
\text { white bread with butter, } \\
2 \text { eggs fried in butter, } 2 \text { slices } \\
\text { of bacon, } 2 \text { ounces of } \\
\text { hash-browned potatoes, and a } \\
\text { glass of whole milk } \\
\text { The low-fat meal: } 30 \mathrm{~g} \\
\text { cornflakes, } 100 \mathrm{~g} \\
\text { semi-skimmed milk, } \\
\text { and } 2 \text { slices of toasted white } \\
\text { bread with margarine and } \\
\text { marmalade }\end{array}$ & $\begin{array}{l}1728 \mathrm{mg} \\
\text { GW433908G (taken } \\
\text { after low- or } \\
\text { high-fat meal), } \\
1200 \mathrm{mg} \\
\text { amprenavir (taken } \\
\text { after low-fat meal) }\end{array}$ & \\
\hline
\end{tabular}


Table 1. Cont.

\begin{tabular}{|c|c|c|c|c|c|c|}
\hline $\begin{array}{l}\text { Author, Year of } \\
\text { Publication }\end{array}$ & Study Setting & Study Design & Study Participants & Food or Dietary Supplements & $\begin{array}{c}\text { Antiretroviral } \\
\text { Drugs }\end{array}$ & Major Outcomes \\
\hline $\begin{array}{l}\text { Piscitelli et al., } \\
2002[28]\end{array}$ & $\begin{array}{l}\text { United States } \\
\text { of America }\end{array}$ & $\begin{array}{l}\text { Two-treatment, } \\
\text { 3-period, } \\
\text { single-sequence, } \\
\text { longitudinal study }\end{array}$ & $\begin{array}{l}9 \text { healthy } \\
\text { subjects, mean age } \\
38 \pm 7.8 \text { years }\end{array}$ & Garlic caplet & $\begin{array}{l}1200 \text { mg saquinavir, } \\
\text { taken together with } \\
\text { garlic }\end{array}$ & $\begin{array}{l}\text { In the presence of garlic, the mean } \\
\text { saquinavir } \mathrm{AUC}_{0-8}, \mathrm{C}_{\text {trough, }} \text { and } \mathrm{C}_{\max } \\
\text { were decreased. }\end{array}$ \\
\hline $\begin{array}{l}\text { Slain et al., } \\
2005 \text { [29] }\end{array}$ & $\begin{array}{l}\text { United States } \\
\text { of America }\end{array}$ & $\begin{array}{l}\text { Prospective, } \\
\text { open-label, } \\
\text { longitudinal, } \\
\text { two-period time } \\
\text { series }\end{array}$ & $\begin{array}{l}7 \text { healthy } \\
\text { subjects, mean age } \\
23.4 \pm 1.6 \text { years }\end{array}$ & $1000 \mathrm{mg}$ vitamin $\mathrm{C}$ & $\begin{array}{l}800 \mathrm{mg} \text { indinavir, } \\
\text { taken at least } 3 \mathrm{~h} \\
\text { separately from } \\
\text { vitamin C }\end{array}$ & $\begin{array}{l}\text { High doses of vitamin } C \text { can significantly } \\
\text { reduce steady-state indinavir plasma } \\
\text { concentrations by } 20 \% \text {. }\end{array}$ \\
\hline $\begin{array}{l}\text { Mouly et al., } \\
2005 \text { [30] }\end{array}$ & $\begin{array}{l}\text { United States } \\
\text { of America }\end{array}$ & $\begin{array}{l}\text { Randomized, } \\
\text { 2-phase, crossover } \\
\text { study }\end{array}$ & $\begin{array}{l}20 \text { healthy } \\
\text { subjects, mean age } \\
28 \pm 9 \text { years }\end{array}$ & $\begin{array}{l}\text { Seville }^{\circledR} \text { orange juice (prepared by } \\
\text { squeezing fruit) }\end{array}$ & $600 \mathrm{mg}$ saquinavir & $\begin{array}{l}\text { Seville }{ }^{\circledR} \text { orange juice delayed absorption of } \\
\text { saquinavir (prolonged } t_{\max } \text { ). }\end{array}$ \\
\hline $\begin{array}{l}\text { DiCenzo et al., } \\
2006 \text { [31] }\end{array}$ & $\begin{array}{l}\text { New York, } \\
\text { United States } \\
\text { of America }\end{array}$ & $\begin{array}{l}\text { Prospective } \\
\text { pharmacokinetic } \\
\text { analysis }\end{array}$ & $\begin{array}{l}10 \text { healthy } \\
\text { subjects, mean age } \\
30.7 \pm 9.4 \text { years }\end{array}$ & $\begin{array}{l}500 \mathrm{mg} \text { quercetin with food } \\
\text { (standardized light breakfast of } 40 \mathrm{~g} \\
\text { of Cheerios }{ }^{\circledR} \text { cereal, } 350 \mathrm{~g} \text { of } 2 \% \\
\text { milk, } 43 \mathrm{~g} \text { of toasted white bread, } \\
9 \mathrm{~g} \text { of margarine, and } 4 \mathrm{~g} \text { of sugar.) }\end{array}$ & $\begin{array}{l}1200 \text { mg saquinavir, } \\
\text { taken together with } \\
\text { quercetin }\end{array}$ & $\begin{array}{l}\text { Administration of quercetin did not } \\
\text { influence plasma saquinavir concentration }\end{array}$ \\
\hline $\begin{array}{l}\text { Sekar et al., } \\
2007[32]\end{array}$ & Belgium & $\begin{array}{l}\text { Open-label, } \\
\text { 2-panel, } \\
\text { randomized, 3-way } \\
\text { crossover study }\end{array}$ & $\begin{array}{l}22 \text { healthy subjects, } \\
\text { median age } 32 \\
(2-50) \text { years males, } \\
49(34-55) \text { years } \\
\text { females }\end{array}$ & 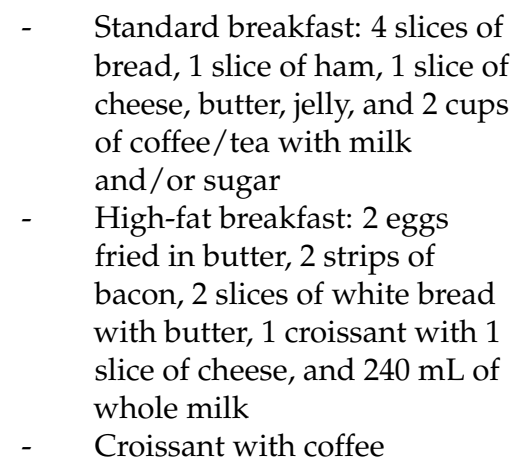 & $\begin{array}{l}100 \mathrm{mg} \text { ritonavir } \\
\text { bd on days } 1 \text { to } 5, \\
\text { with a single } \\
400 \mathrm{mg} \text { darunavir } \\
\text { given on day } 3 \\
\text { (darunavir/ritonavir), } \\
\text { taken immediately } \\
\text { after meal }\end{array}$ & $\begin{array}{l}\text { Administration of darunavir/ritonavir in a } \\
\text { fasting state resulted in a decrease in } \\
\text { darunavir } C_{\max } \text { and } A U C_{\text {last }} \text { of } \\
\text { approximately } 30 \% \text { compared with } \\
\text { administration after a standard meal. }\end{array}$ \\
\hline
\end{tabular}


Table 1. Cont.

\begin{tabular}{|c|c|c|c|c|c|c|}
\hline $\begin{array}{l}\text { Author, Year of } \\
\text { Publication }\end{array}$ & Study Setting & Study Design & Study Participants & Food or Dietary Supplements & $\begin{array}{c}\text { Antiretroviral } \\
\text { Drugs }\end{array}$ & Major Outcomes \\
\hline $\begin{array}{l}\text { Robertson et al., } \\
\quad 2008 \text { [33] }\end{array}$ & $\begin{array}{l}\text { United States } \\
\text { of America }\end{array}$ & $\begin{array}{l}\text { Single-center, } \\
\text { open-label }\end{array}$ & $\begin{array}{l}14 \text { healthy } \\
\text { subjects, mean age } \\
29.5(23-48) \text { years }\end{array}$ & $120 \mathrm{mg}$ Ginkgo biloba & $\begin{array}{l}400 \mathrm{mg} \\
\text { lopinavir } / 100 \mathrm{mg} \\
\text { ritonavir, taken } \\
\text { together with } \\
\text { Ginkgo biloba } \\
\text { extract }\end{array}$ & $\begin{array}{l}\text { Neither lopinavir nor ritonavir } \\
\text { pharmacokinetic parameters were } \\
\text { significantly changed by } 2 \text { weeks of Ginkgo } \\
\text { biloba extract coadministration. }\end{array}$ \\
\hline $\begin{array}{l}\text { Patel et al., } \\
2011 \text { [34] }\end{array}$ & - & $\begin{array}{l}\text { Open-label, } \\
\text { randomized, } \\
\text { crossover study }\end{array}$ & $\begin{array}{l}16 \text { healthy } \\
\text { subjects, mean age } \\
30.8 \text { years }\end{array}$ & $\begin{array}{l}\text { Multivitamin tablet ( } 162 \mathrm{mg} \text { of } \\
\text { elemental calcium and } 100 \mathrm{mg} \text { of } \\
\text { magnesium per tablet, in addition } \\
\text { to iron, zinc, and copper) }\end{array}$ & $\begin{array}{l}50 \mathrm{mg} \\
\text { S/GSK1349572, } \\
\text { taken together with } \\
\text { multivitamin tablet }\end{array}$ & $\begin{array}{l}\text { Multivitamins did not significantly affect } \\
\text { S/GSK1349572 pharmacokinetics. } \\
\text { Therefore, they may be co-administered. }\end{array}$ \\
\hline $\begin{array}{l}\text { Calderõn et al., } \\
2014 \text { [35] }\end{array}$ & $\begin{array}{l}\text { United States } \\
\text { of America }\end{array}$ & $\begin{array}{l}\text { Single sequence, } \\
\text { open-label, } \\
\text { single-center } \\
\text { pharmacokinetic } \\
\text { investigation }\end{array}$ & $\begin{array}{l}12 \text { healthy subjects, } \\
\text { median age } 32 \\
(23-42) \text { years }\end{array}$ & $500 \mathrm{mg}$ Panax ginseng & $\begin{array}{l}400 \mathrm{mg} \\
\text { lopinavir } / 100 \mathrm{mg} \\
\text { ritonavir, taken } \\
\text { together with } \\
\text { Panax ginseng }\end{array}$ & $\begin{array}{l}\text { Neither lopinavir nor ritonavir } \\
\text { pharmacokinetic parameters were } \\
\text { changed by two weeks of Panax ginseng } \\
\text { administration. }\end{array}$ \\
\hline $\begin{array}{l}\text { Song et al., } \\
2015 \text { [36] }\end{array}$ & $\begin{array}{l}\text { United States } \\
\text { of America }\end{array}$ & $\begin{array}{l}\text { Open-label, } \\
\text { randomized, } \\
\text { 2-cohort, 4-period } \\
\text { crossover study }\end{array}$ & $\begin{array}{l}21 \text { healthy } \\
\text { subjects, mean age } \\
33.2 \text { years }\end{array}$ & $\begin{array}{l}1200 \mathrm{mg} \text { Calcium carbonate/324 mg } \\
\text { Ferrous fumarate }\end{array}$ & $\begin{array}{l}50 \text { mg dolutegravir, } \\
\text { taken together with } \\
\text { Calcium or iron } \\
\text { supplements }\end{array}$ & $\begin{array}{l}\text { During mealtime, dolutegravir and } \\
\text { calcium or iron supplements can be } \\
\text { co-administered since the food } \\
\text { increases the exposure. } \\
\text { Under fasted conditions, dolutegravir } \\
\text { should be administered } 2 \mathrm{~h} \text { before or } \\
6 \mathrm{~h} \text { after administration of calcium or } \\
\text { iron supplements, as there is a } \\
\text { reduction in } \mathrm{AUC}_{0-\infty}, \mathrm{C}_{\max } \text {, and } \mathrm{C}_{24} \\
\text { of dolutegravir by chelation. }\end{array}$ \\
\hline $\begin{array}{l}\text { Buchanan et al., } \\
\text { 2017 [37] }\end{array}$ & $\begin{array}{l}\text { United States } \\
\text { of America }\end{array}$ & $\begin{array}{l}\text { Phase 1, } \\
\text { single-center, } \\
\text { randomized, } \\
\text { open-label, } \\
\text { 5-period } \\
\text { crossover study }\end{array}$ & $\begin{array}{l}15 \text { healthy } \\
\text { subjects, mean age } \\
39.8 \pm 12.5 \text { years }\end{array}$ & $\begin{array}{l}\text { High-mineral content water } \\
\left(\text { Contrex }{ }^{\circledR} \text { : calcium } 468 \mathrm{mg} / \mathrm{L} \text {, }\right. \\
\text { magnesium } 74.5 \mathrm{mg} / \mathrm{L}), \\
\text { Low-mineral content water ( } 5 \% \\
\text { Contrex }{ }^{\circledR} \text { in purified water) }\end{array}$ & $\begin{array}{l}20 \mathrm{mg} \text { dolutegravir } \\
\text { (dispersed in } \\
12.5 \mathrm{~mL} \text { of high- or } \\
\text { low-mineral water }\end{array}$ & $\begin{array}{l}\text { Dolutegravir pharmacokinetic } \\
\text { parameters were unaffected by mineral } \\
\text { contents in water. }\end{array}$ \\
\hline
\end{tabular}


Table 1. Cont.

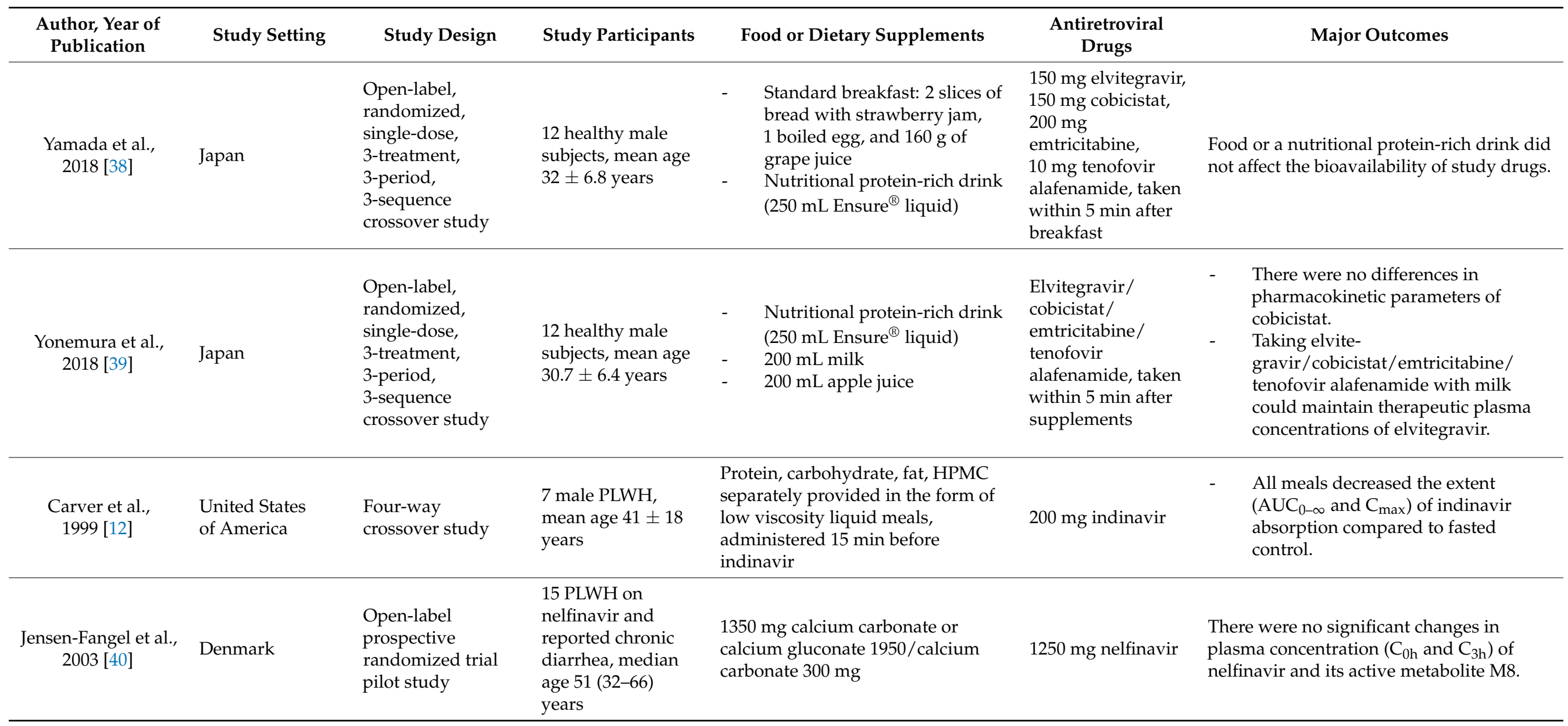


Table 1. Cont.

\begin{tabular}{|c|c|c|c|c|c|c|}
\hline $\begin{array}{l}\text { Author, Year of } \\
\text { Publication }\end{array}$ & Study Setting & Study Design & Study Participants & Food or Dietary Supplements & $\begin{array}{c}\text { Antiretroviral } \\
\text { Drugs }\end{array}$ & Major Outcomes \\
\hline $\begin{array}{l}\text { Roberts et al., } \\
2011 \text { [15] }\end{array}$ & $\begin{array}{l}\text { United States } \\
\text { of America }\end{array}$ & Case report & $\begin{array}{l}\text { An HIV-infected } \\
\text { man }\end{array}$ & $\begin{array}{l}\text { Calcium carbonate } 1 \mathrm{~g} \text { vitamin D3 } \\
400 \mathrm{IU} \text { (cholecalciferol) }\end{array}$ & $\begin{array}{l}400 \mathrm{mg} \\
\text { raltegravir/200 mg } \\
\text { emtricitabine } \\
+300 \mathrm{mg} \text { tenofovir } \\
\text { disoproxil } \\
\text { fumarate }\end{array}$ & $\begin{array}{l}\text { After } 10 \text { months of starting } \\
\text { raltegravir, the patient subsequently } \\
\text { developed detectable HIV-1 RNA } \\
\text { levels (7190 copies/mL) with } \\
\text { documented resistance to raltegravir. } \\
\text { Calcium supplements may lead to } \\
\text { subtherapeutic raltegravir levels due } \\
\text { to binding to the divalent metal } \\
\text { ion-chelating motif of raltegravir. }\end{array}$ \\
\hline $\begin{array}{c}\text { Sheehan et al., } \\
2012 \text { [41] }\end{array}$ & Canada & $\begin{array}{l}\text { Multi-center, } \\
\text { open-label, } \\
\text { non-randomized } \\
\text { steady-state } \\
\text { pharmacokinetic } \\
\text { study }\end{array}$ & $\begin{array}{l}11 \text { PLWH receiving } \\
\text { nelfinavir } 1250 \mathrm{mg} \\
\text { twice daily with at } \\
\text { least two NRTIs for } \\
\text { at least two weeks, } \\
\text { mean age } \\
45.5 \pm 9.4 \text { years }\end{array}$ & $\begin{array}{l}\beta \text {-carotene } 25,000 \mathrm{IU} \text {, taken together } \\
\text { with nelfinavir }\end{array}$ & $1250 \mathrm{mg}$ nelfinavir & $\begin{array}{l}\text { - } \quad \beta \text {-carotene supplementation did not } \\
\text { cause any clinically significant } \\
\text { difference in the nelfinavir and M8 } \\
\text { exposure. } \\
\text { Mean CD4\% and CD4: CD8 ratio } \\
\text { increased significantly. }\end{array}$ \\
\hline $\begin{array}{c}\text { Abdissa et al., } \\
2015 \text { [42] }\end{array}$ & Ethiopia & $\begin{array}{l}\text { Double-blinded, } \\
\text { randomized trial }\end{array}$ & $\begin{array}{l}282 \text { ART-naïve } \\
\text { PLWH, mean age } \\
32.2 \pm 8.0 \text { years } \\
\text { (LNS/w), } 34.5 \pm \\
10.3 \text { years (LNS/s), } \\
31.7 \pm 8.5 \text { years (no } \\
\text { LNS) }\end{array}$ & $\begin{array}{l}\text { Lipid-based nutrient supplement } \\
\text { containing whey (LNS/w) } \\
\text { Lipid-based nutrient supplement } \\
\text { containing soy (LNS/s) }\end{array}$ & $\begin{array}{l}600 \mathrm{mg} \\
\text { efavirenz/200 mg } \\
\text { nevirapine }\end{array}$ & $\begin{array}{l}\text { - LNS intake was associated with lower } \\
\text { plasma nevirapine trough } \\
\text { concentrations, indicating possible } \\
\text { drug-LNS interactions. } \\
\text { LNS did not affect EFV trough } \\
\text { concentrations. }\end{array}$ \\
\hline
\end{tabular}


Table 1. Cont.

\begin{tabular}{|c|c|c|c|c|c|c|}
\hline $\begin{array}{l}\text { Author, Year of } \\
\text { Publication }\end{array}$ & Study Setting & Study Design & Study Participants & Food or Dietary Supplements & $\begin{array}{l}\text { Antiretroviral } \\
\text { Drugs }\end{array}$ & Major Outcomes \\
\hline $\begin{array}{c}\text { Munkombwe et al., } \\
2016 \text { [43] }\end{array}$ & Zambia & $\begin{array}{l}\text { Randomized } \\
\text { controlled trial }\end{array}$ & $\begin{array}{l}130 \text { ART-naïve } \\
\text { malnourished } \\
\text { PLWH }(\mathrm{BMI}< \\
\left.18 \mathrm{~kg} / \mathrm{m}^{2}\right), \text { mean } \\
\text { age } 35 \pm 8 \text { years } \\
(\mathrm{LNS}), 38 \pm 9 \text { years } \\
\text { (LNS-VM) }\end{array}$ & $\begin{array}{l}\text { Lipid-based nutrient supplements } \\
\text { (LNS) or LNS with vitamins and } \\
\text { minerals (LNS-VM) }\end{array}$ & $\begin{array}{l}300 \mathrm{mg} \text { tenofovir } \\
\text { disoproxil } \\
\text { fumarate } / 200 \mathrm{mg} \\
\text { emtricitabine } / 600 \\
\text { mg efavirenz }\end{array}$ & $\begin{array}{l}\text { The LNS-VM regimen appeared to offer } \\
\text { protection against phosphate and } \\
\text { potassium loss during HIV/AIDS } \\
\text { treatment. }\end{array}$ \\
\hline
\end{tabular}




\subsection{Studies Included in the Qualitative Analysis}

Two-hundred and seventy-nine healthy subjects, and five-hundred and six PLWH who participated in twenty-four studies were included in the qualitative analysis (Table 1). The participants include various ethnic and racial groups such as people of European, African, and Asian descent. There were 3 randomized controlled trials, 13 cross-over studies, 2 longitudinal studies, 4 pharmacokinetic studies, 1case report, and 1cross-sectional study. Among the included studies, three studies reported the effects of grapefruit juice or Seville orange juice on ARV pharmacokinetics $[24,26,30]$. While nine studies investigated the effects of food or nutrient supplement on ARV pharmacokinetics [12,14,25,27,32,38,42-44]. The rest of the studies explored the pharmacokinetic interactions between ARV and vitamins or minerals $[15,29,34,36,37,40]$, and dietary supplements [28,31,33,35,39,41]. Most studies showed non-significant changes in ARV pharmacokinetic parameters when coadministered with meals. However, there was a significant decrease in the extent of absorption of indinavir with liquid meals reported by Carver et al. [12]; protein, carbohydrate, fat, and viscosity meal treatments reduced indinavir $\mathrm{AUC}_{0}-\infty$ by $68 \%, 45 \%, 34 \%$, and $30 \%$, respectively, while these meals decreased $\mathrm{C}_{\max }$ by $74 \%, 59 \%, 46 \%$, and $36 \%$, respectively. Regarding the meal compositions, fat meals resulted in the highest variability on the plasma indinavir levels. The consumption of garlic supplement decreased AUC and $\mathrm{C}_{\max }$ of saquinavir by $51 \%$ and $54 \%$, respectively [28], while other dietary supplements such as quercetin, Ginkgo extract, Ginseng extract, $\beta$-carotene did not significantly affect saquinavir, lopinavir, ritonavir, and nelfinavir pharmacokinetics [31,33,35,41]. Grapefruit juice administration increased the oral bioavailability of saquinavir [24] while its concurrent intake did not lead to any change in indinavir concentrations [26]. A pharmacokinetic study by Slain et al. [29] indicated that after a week of $1000 \mathrm{mg}$ vitamin C supplementation, the steady-state indinavir plasma concentration was reduced by $20 \%$. On the contrary, some studies have reported that the use of multivitamins or minerals did not lead to any pharmacokinetic variation in ARV such as dolutegravir and nelfinavir [34,36,37,40]. In a case report of an HIV-infected man receiving raltegravir, concomitant calcium administration resulted in virologic failure showing detectable plasma HIV-1 RNA levels [15]. Coadministration of protease inhibitors such as nelfinavir with $\beta$-carotene led to a significant increase not only in mean CD4\%, but also in the CD4:CD8 ratio in PLWH in one study [41]. In addition, the study also found an increase in CD4 counts; although, it was not clinically significant.

\subsection{Studies Included in the Meta-Analysis}

A total of 122 participants from 5 studies were included in the meta-analysis. Studies by Moore et al. [14], Yuen et al. [25], Falcoz et al. [27], Sekar et al. [32], and Yamada et al. [38] reported the pharmacokinetic outcomes of the interaction between food and ARV were eligible for meta-analysis. In general, the heterogeneity was high, and the $95 \%$ confidence intervals of the pooled data were close to the most weighted and included studies whose intervals were narrow.

Figure 2 shows the meta-analysis of the pharmacokinetic effects of food on the $\mathrm{AUC}_{\text {inf }}$ of ARV. There were no significant changes in $\mathrm{AUC}_{\text {inf }}$ of $\mathrm{ARV}$ between fasted and fed conditions (mean difference $-24.64,95 \%$ CI -141.34to 92.07, $p=0.68$ ), with a substantial heterogeneity of $75 \%$. 


\begin{tabular}{|c|c|c|c|c|c|c|c|c|c|c|c|}
\hline Study or Subgroup & \multicolumn{3}{|c|}{$\begin{array}{c}\text { Fed } \\
\end{array}$} & \multicolumn{3}{|c|}{$\begin{array}{l}\text { Fasted } \\
\text { Mean [ng.h/mL] SD } \quad \text { [ng } \cdot h / m L] \quad \text { Total }\end{array}$} & \multicolumn{3}{|c|}{ Mean Difference } & \multirow{2}{*}{\multicolumn{2}{|c|}{$\begin{array}{l}\text { Mean Difference } \\
\text { IV, Random, } 95 \% \mathrm{Cl}\end{array}$}} \\
\hline Moore 1999 3TC combined tablet & $6,035.4$ & 1160.6 & 24 & 6.1376 & & & & & & & \\
\hline Moore 1999 AZT combined tablet & 21476 & 6646 & 24 & 23982 & & 24 & & $-250.60[-638.46,137.26]$ & & & \\
\hline Yuen 2001 3TC combined tablet & 5,610 & 1,340 & 24 & 6,060 & 1,370 & $\begin{array}{l}24 \\
24\end{array}$ & 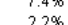 & $-250.60[-638.46,131.26]$ & 19999 & & \\
\hline Yuen $2001 \mathrm{ABC}$ combined tablet & 6,570 & 2110 & 24 & 7310 & 2710 & 24 & $0.2 \%$ & $-450.00[-1216.70,316.70]$ & 2001 & & \\
\hline Yuen 2001 AZT combined tablet & 2,050 & 540 & 24 & 2,070 & 720 & 24 & $83 \%$ & $-740.00[-2714.08,634.007$ & 2001 & $\leftarrow$ & \\
\hline Yamada 2018 TAF & 154 & 33 & 12 & 141 & 42 & 12 & $39.4 \%$ & $13.00[-17.22,43.22]$ & 2018 & & - \\
\hline Yamada 2018 TDF & 288 & 50 & 12 & 279 & 52 & 12 & $38.6 \%$ & $9.00[-31.82,49.82]$ & 2018 & & \\
\hline Yamada $2018 \mathrm{COBI}$ & 6,851 & 2,874 & 12 & 7,715 & 3,036 & 12 & $0.2 \%$ & $-864.00[-3229.34,1501.34]$ & 2018 & & \\
\hline Yamada 2018 EVG & 32,267 & 7,889 & 12 & 17,111 & 5,128 & 12 & $0.0 \%$ & $15156.00[9832.35,20479.65]$ & 2018 & & ' \\
\hline Yamada $2018 \mathrm{FTC}$ & 14,193 & 2,751 & 12 & 14,295 & 2,803 & 12 & $0.3 \%$ & $-102.00[-2324.12,2120.12]$ & 2018 & & \\
\hline Total $(95 \% \mathrm{Cl})$ & & & 180 & & & 180 & $100.0 \%$ & $-24.64[-141.34,92.07]$ & & & \\
\hline $\begin{array}{l}\text { Heterogeneity: Tau }=8752.71 ; C h \\
\text { Test for overall effect: } Z=0.41(\mathrm{P}=\end{array}$ & $\begin{array}{l}=36.06 \\
1.68)\end{array}$ & & & & & & & & & $\begin{array}{l}50 \\
A \cup C \text { in } f a s\end{array}$ & 100 \\
\hline
\end{tabular}

Figure 2. Forest plot showing the mean difference in $\mathrm{AUC}_{\text {inf }}$ of $\mathrm{ARV}$ under fasted and fed states (3TC, lamivudine; $\mathrm{ABC}$, abacavir; AZT, zidovudine; COBI, cobicistat; EVG, elvitegravir; FTC, emtricitabine; TAF, tenofovir alafenamide; TDF, tenofovir).

The overall effect of food on $\mathrm{C}_{\max }$ of NRTI group shows no clinical significance between fasted and fed conditions (mean difference $-0.56,95 \%$ CI -2.31 to $1.20, p=0.53$ ) in fixed effect models, while $\mathrm{C}_{\text {max }}$ of NRTI group shows a significant decrease during fed state (mean difference $-204.70,95 \%$ CI -337.14 to $-72.26, p=0.002$ ) in random effect models with the heterogeneity value of $84 \%$. Interestingly, there was a significant increase in the $C_{\max }$ of PI group during fed state (mean difference $845.51(110.10-1580.92, p=0.02)$ as shown in Figure 3. Figure 4 indicates the overall effect of food on $\mathrm{C}_{24}$, which was not significant (mean difference $-0.13,95 \% \mathrm{CI}-3.08$ to $2.82, p=0.93$ ).

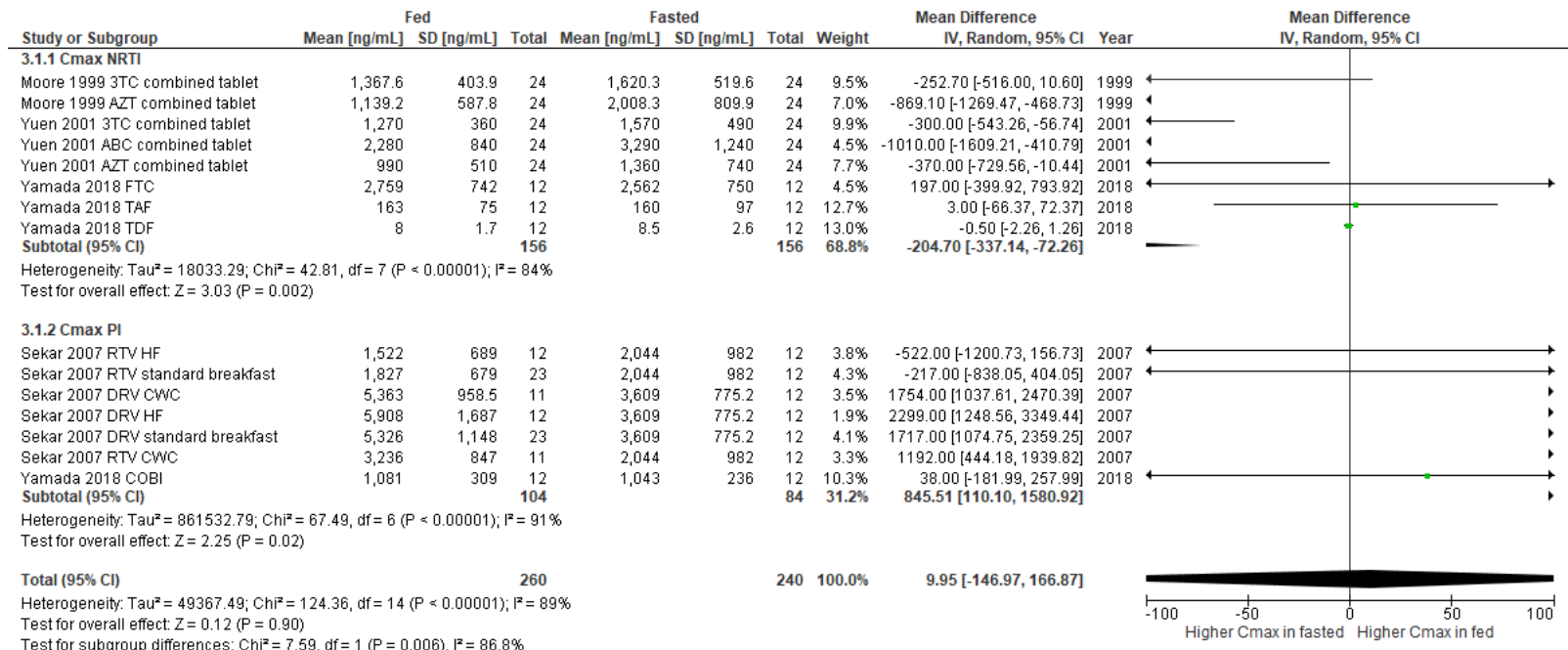

Figure 3. Forest plot showing the mean difference in $\mathrm{C}_{\max }$ of ARV under fasted and fed states by ARV regimens (3TC, lamivudine; $\mathrm{ABC}$, abacavir; $\mathrm{AZT}$, zidovudine; $\mathrm{COBI}$, cobicistat; $\mathrm{CWC}$, croissant with coffee; DRV, darunavir; FTC, emtricitabine; HF, high fat; NRTI, nucleoside reverse transcriptase inhibitor; PI, protease inhibitor; RTV, ritonavir; TAF, tenofovir alafenamide; TDF, tenofovir).

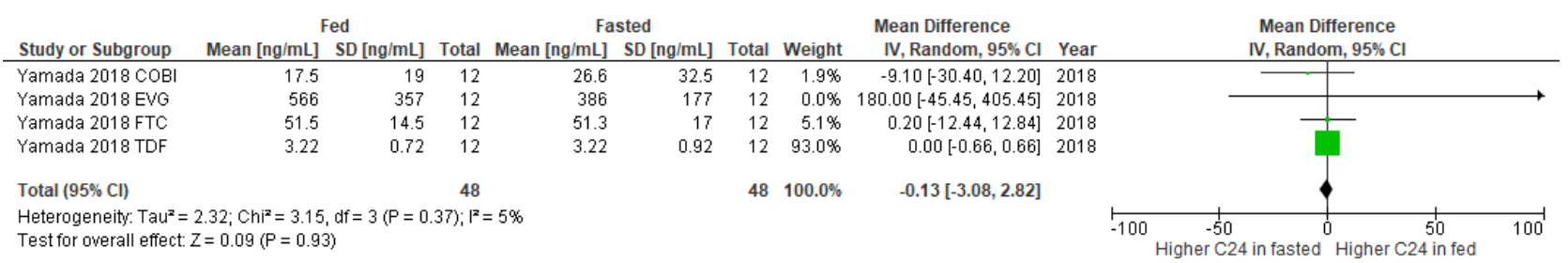

Figure 4. Forest plot showing the mean difference in $\mathrm{C}_{24}$ of ARV under fasted and fed states (COBI, cobicistat; EVG, elvitegravir; FTC, emtricitabine; TDF, tenofovir). 
The results of the subgroup meta-analysis of pharmacokinetic changes in $t_{\text {max }}$ of ARV depending upon food or supplement administration are shown in Figure 5. The overall effect in NRTI group indicates a significantly higher $t_{\max }$ value under fed condition (mean difference $0.67,95 \%$ CI $0.44-0.91, p<0.00001$ ), showing the heterogeneity of $63 \%$. Similarly, we found a significant increase in $t_{\max }$ in fed state compared to fasted condition in PI receiving groups (mean difference 1.29, 95\% CI 0.81-1.77, $p<0.00001$ ) (Figure 5). For both NRTI and PI groups, none of these show any clinically significant changes in $t_{1 / 2}$ (Figure 6). We observed no statistical heterogeneity on $t_{1 / 2}$ of both NRTI and PI regimens.

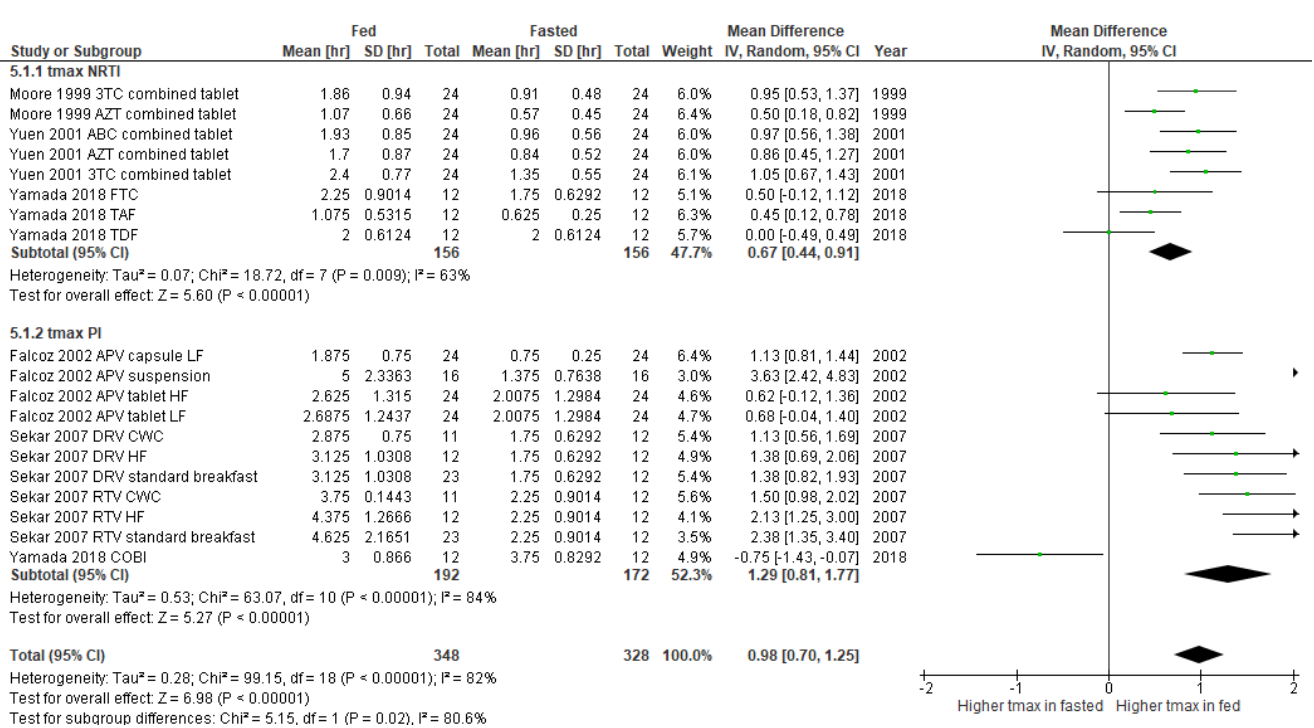

Figure 5. Forest plot showing the mean difference in $t_{\max }$ of $A R V$ under fasted and fed states by ARV regimens (3TC, lamivudine; ABC, abacavir; APV, amprenavir; AZT, zidovudine; COBI, cobicistat; CWC, croissant with coffee; DRV, darunavir; FTC, emtricitabine; HF, high fat; LF, low fat; NRTI, nucleoside reverse transcriptase inhibitor; PI, protease inhibitor, RTV, ritonavir; TAF, tenofovir alafenamide; TDF, tenofovir).

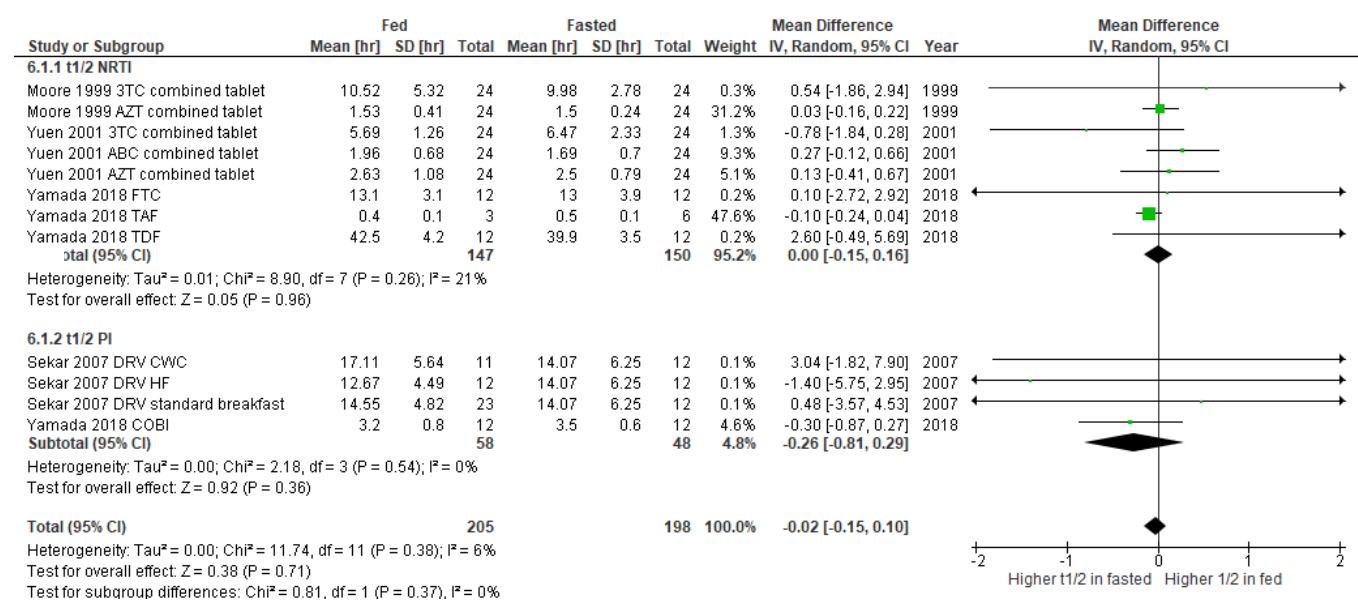

Figure 6. Forest plot showing the mean difference in $t_{1 / 2}$ of ARV under fasted and fed states by ARV regimens (3TC, lamivudine; ABC, abacavir; AZT, zidovudine; COBI, cobicistat; CWC, croissant with coffee; DRV, darunavir; FTC, emtricitabine; HF, high fat; NRTI, nucleoside reverse transcriptase inhibitor; PI, protease inhibitor; TAF, tenofovir alafenamide; TDF, tenofovir).

\subsection{Publication Bias}

Publication bias was assessed by visual inspection of the funnel plot, which is shown in Figure 7 . It was found that there were seven missing studies for $t_{\max }$. The distribution of the standard error was close to the peak of the funnel plot, which demonstrated that 
there may be systematic difference or publication bias between smaller and larger studies. Further statistical test for the funnel plot asymmetry was not performed since the forest plot that had the largest number of included studies included only five studies [45].

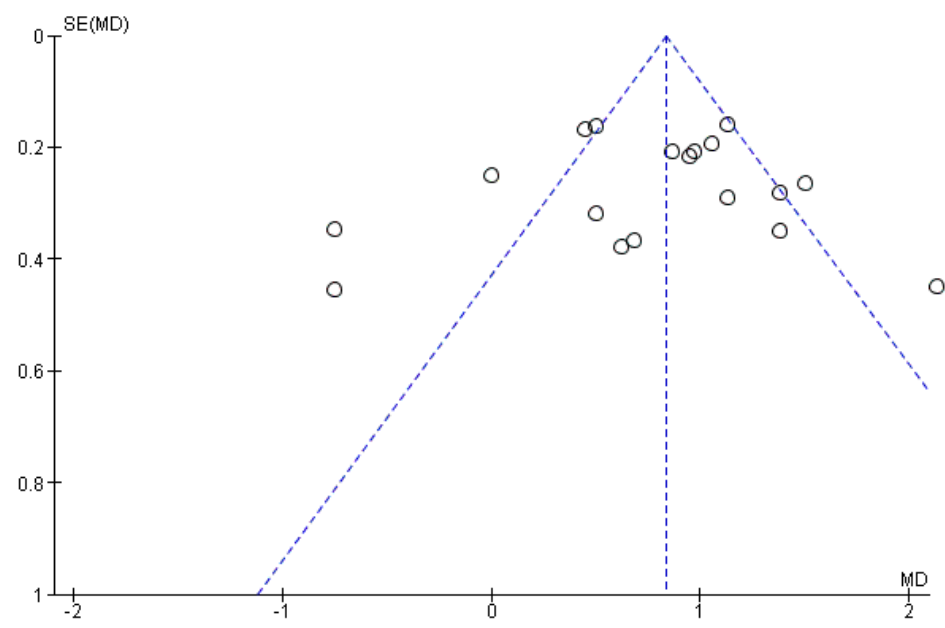

Figure 7. Funnel plot of the adjusted association between $t_{\max }$ and food (SE, standard error; MD, mean difference).

\section{Discussion}

This systematic review and meta-analysis mainly presented no significant pharmacokinetic changes in AUC, $\mathrm{C}_{24}$, and $\mathrm{t}_{1 / 2}$ of $\mathrm{ARV}$ when co-administered with food or dietary supplements. However, it can be highlighted that there was an increase in $t_{\max }$ of NRTI and PI under fed conditions. Of interest, the higher $\mathrm{C}_{\max }$ in fed state was observed in participants receiving the PI regimen in this study. Among the articles included in this systematic review, most of the ARV's pharmacokinetic parameters were not clinically altered by the concurrent administration of food and other supplements such as quercetin, Ginkgo extract, vitamin supplement, Ginseng, or $\beta$-carotene.

The different classes of ARV have varied pharmacokinetic metabolisms. PIs are extensively metabolized by the cytochrome P (CYP) 450 enzymes in the liver and small intestine [46]. Thus, ingestion of some foods or juices that have an inhibitory effect on CYP450 may increase concentrations of PIs. Kupferschmidt et al. [24] reported an increase in the bioavailability of saquinavir bioavailability after an intake of grapefruit juice, which is widely known as a CYP3A4 inhibitor [47]. In addition, a previous study reported an increase in AUC of atazanavir, lopinavir/ritonavir, nelfinavir, and saquinavir when ingested with food [48]. Under the fed condition, there was a long $t_{\max }$ of amprenavir from 1 to $4 \mathrm{~h}$ compared with the fasting state [27]. The $t_{\max }$ of darunavir under fasted conditions was approximately $1.5 \mathrm{~h}$, whereas it was increased to 3-4 $\mathrm{h}$ after administration with food including standard breakfast, croissant with coffee, and high-fat breakfast. Similarly, the median $t_{\max }$ of $2 \mathrm{~h}$ of ritonavir without food extended to 4 or $5 \mathrm{~h}$ when food was administered concurrently [32]. Regarding the changes in plasma concentration of PI such as darunavir, Sekar et al. [32] reported the increased $\mathrm{C}_{\max }$ by about $30 \%$ under fed condition compared with the fasted state. Another class of ARV, NNRTIs such as efavirenz and nevirapine are also metabolized by several liver CYP isoenzymes [49]. The administration of efavirenz with high fat or high caloric meal was associated with the increased mean AUC by $28 \%$ and mean $\mathrm{C}_{\max }$ by $29 \%$, respectively, compared to fasted conditions [50]. Most NRTIs are degraded by liver enzymes from the purine or pyrimidine nucleoside salvage pathway, depending upon the NRTI analogs [51]. Since they are not extensively metabolized by CYP450 [51], they have less interaction with food or other drugs. The delayed absorption of abacavir, lamivudine, and zidovudine was noted by Yuen et al. [25] an hour later in median $t_{\max }$ under fed conditions compared to fasted conditions. Similarly, Moore et al. [14] observed the slower $t_{\max }$ of lamivudine and zidovudine by $30 \mathrm{~min}$ and $45 \mathrm{~min}$, 
respectively, after the administration of combined tablets with a high-fat meal. The mean $t_{\max }$ of emtricitabine and tenofovir alafenamide under standard breakfast was $2 \mathrm{~h}$ and $1 \mathrm{~h}$, respectively, which was longer than those under fasted conditions [38,52]. The concurrent administration of integrase inhibitors (IIs) and polyvalent cations such as magnesium and calcium should be monitored since these cations may bind with ARV, which leads to decreased plasma levels. Although Buchanan et al. [37] reported no clinical significance, dolutegravir, according to its medication package insert, should be taken $2 \mathrm{~h}$ before or $6 \mathrm{~h}$ after administering a cation containing antacids or laxatives, sucralfate, oral iron supplements, oral calcium supplements, or buffered medications [36,53].

The impacts of vitamin and mineral supplements on ARVs were mostly investigated in IIs [15,34,36,37] and some PIs [29,40]. Co-administration of $1200 \mathrm{mg}$ calcium carbonate and $324 \mathrm{mg}$ ferrous fumarate with dolutegravir during fasted conditions decreased in $\mathrm{AUC}_{0-\infty}$, $\mathrm{C}_{\max }$, and $\mathrm{C}_{24}$ of dolutegravir by respective $37 \%$ to $39 \%$ and $54 \%$ to $57 \%$ due to chelation with polyvalent cations [36]. However, coadministration of these divalent cations and dolutegravir under fed conditions counteracted such the interaction [36]. Moreover, Roberts et al. [15] reported subtherapeutic raltegravir levels when co-administered with $1000 \mathrm{mg}$ calcium carbonate. Nonetheless, information on the time interval between raltegravir and calcium intake or whether they were administered under fed or fasted conditions were not reported. Given that calcium supplement is necessary for the prevention of osteoporosis, especially in PLWH [54], future studies investigating the magnitude of such interaction between raltegravir and calcium supplement under fasted and fed conditions should be conducted. If the fed condition can counteract the interaction, calcium supplements may be co-administered with raltegravir. Concerning the impacts of vitamin and mineral supplements on PIs, while the divalent calcium ion did not significantly alter nelfinavir and its active metabolite M8 concentrations [40], co-administration with vitamin C significantly decreased steady-state indinavir concentrations [15]. This could be explained by the inductive property of vitamin C on CYP isoenzymes observed in animal studies [55-59]. However, another study reported no significant effect of vitamin C on CYP3A4, the primary enzyme used to metabolize indinavir activity [60]. Based on the contradictory effects of vitamin C on CYP3A4, further studies are needed to confirm the results.

Concerning the impacts of various dietary supplements, different classes of ARVs were investigated, mostly the PIs. Piscitelli et al. [28] reported that garlic supplement significantly decreased the extent of saquinavir absorption, as indicated by the decrease in $\mathrm{AUC}_{0-8}$, $\mathrm{C}_{\text {trough, }}$ and $\mathrm{C}_{\max }$. No definite underlying mechanism could be drawn from this study, but the authors proposed that it could be due to the induction of CYP450, or P-glycoprotein (P-gp) produced by long-term use of garlic. Nonetheless, evidence has shown that the effects of garlic on CYP3A4 are controversial as some in vitro studies reported that garlic had an inhibitory effect on CYP3A4 [61]. While other studies showed no significant effect of garlic on CYP3A4 [62,63]. Given that garlic supplements might have a negative effect on saquinavir exposure, garlic supplementation should be avoided in patients receiving treatment with saquinavir.

Quercetin, an inhibitor of various CYP450 including CYP3A4 as well as P-gp, could theoretically be used as a booster of saquinavir levels [64,65]. However, DiCenzo et al. [31] did not find significant effects of quercetin on saquinavir concentrations. The nonsignificant effect of quercetin could partially be explained by the intersubject and intrasubject variability. Given that only ten subjects were included in the study by DiCenzo et al. [31], further studies are required to confirm such results.

Ginkgo biloba, another widely used dietary supplement, has been shown to induce CYP3A activity [66,67]. However, Robertson et al. [33] reported no significant effects of Ginkgo biloba extract on lopinavir and ritonavir pharmacokinetics. These could be explained by the inhibitory effect of co-administered ritonavir on CYP3A. Nevertheless, the impacts of Ginkgo biloba extract on un-boosted protease inhibitors were not investigated in that study, and hence coadministration of Ginkgo biloba extract with un-boosted PI is not theoretically recommended. Similar to the effect of Ginkgo biloba, Panax ginseng has shown an inductive 
effect on CYP3A, which in turn may reduce PI concentrations [68]. However, the two-week administration of Panax ginseng did not alter lopinavir and ritonavir pharmacokinetics [35]. The same reason concerning the inhibitory effect of concurrently administered ritonavir could be applied here. Therefore, a similar recommendation on the use of Panax ginseng and un-boosted PI to that of Ginkgo biloba extract is proposed.

Inconclusive effects of $\beta$-carotene on CYP3A4 have been reported from in vitro studies, as one study found an inhibitory effect [69], while another reported an inductive property [70]. A clinical study investigating the impacts of $\beta$-carotene supplementation at the dose of 25,000 IU twice daily on nelfinavir and its active metabolite M8, which is metabolized by respective CYP2C19 and CYP3A4, indicated no clinically significant effect [41]. This implies that PLWH receiving ARVs that are substrates of CYP2C19 or CYP3A4 may be able to use $\beta$-carotene as a dietary supplement. However, it should be noted that these findings were based only on $15 \mathrm{HIV}$-infected subjects. Further prospective studies investigating the impact of $\beta$-carotene on other CYP2C19 and CYP3A4 substrates may be warranted.

This study has some limitations. The included studies did not report the component of fruit juices, i.e., whether the juice contained pulp or additives. This information may be crucial since these compositions might not be pharmaceutically inert. Since most studies were conducted with different ARV drugs and different supplements, only limited studies were included for meta-analysis. Only food (meal) and ARV pharmacokinetic interaction in healthy subjects were analyzed due to the limited number of studies with supplements and ARV pharmacokinetic interaction. Additionally, caution should be taken when the stratified results are interpreted as the minimal number of studies for subgroup analysis should be more than 10 [71]. Some studies from the qualitative analysis could not be included in the meta-analysis because of the diverse types of supplements among studies. The number of participants in each study was relatively small, and there may also have been interpatient or intrapatient variability. Furthermore, most studies were conducted in different regions with different study designs. Moreover, our systematic review may not be generalizable to all nutrient or food supplements as we did not focus on some products, e.g., probiotics or prebiotics, which may have effects on ARV pharmacokinetics. Of note, the heterogeneity across studies may also affect the results due to the potential effects of some factors such as gender, comorbidities, food compositions, dosage regimen, and duration of treatment.

This study found new information on the potential impact of supplement use on ARV pharmacokinetics. The study highlighted the decreased absorption of NRTIs such as abacavir, emtricitabine, and PIs such as ritonavir and darunavir after co-administration with food. The increased $t_{\max }$ and plasma concentration of some ARV such as darunavir due to ARV-food interaction can suggest nutrition monitoring in relation to HIV and ARV treatment. As a further matter, due to the diversity of supplements, further research that considers not only the variability of interactions but the likelihood of an individual patient to develop potential outcomes should be examined. Improving awareness of therapeutic monitoring followed by pharmacokinetic evaluation of specific interaction should be implemented because there is extensive use of supplements in the HIV population.

\section{Conclusions}

This study pointed out the delayed absorption of NRTIs such as abacavir, emtricitabine, lamivudine, zidovudine, tenofovir alafenamide, and PIs including amprenavir, darunavir, and ritonavir under fed conditions including low-fat and high-fat meals. The higher plasma concentration of PI such as darunavir and cobicistat was also observed in a fed state. Considering the effects of various products on drug-metabolizing enzymes, more evidence in pharmacokinetic fields is required. So far, it is still necessary to investigate the potential interactions of ARV and particular supplement or complementary products in PLWH. Further pharmacokinetic studies are highly recommended to explore the potential pharmacokinetic interaction between food and ARV that might affect therapeutic outcomes. Physicians and healthcare providers need to be aware of the potential interaction between prescribed ARV 
and any mineral supplements that may lead to virologic failure or delayed absorption due to chelation. Since even a small change in plasma drug level can affect therapeutic efficacy, PLWH on ARV should be educated or given information on supplement use.

Supplementary Materials: The following are available online at https:/ /www.mdpi.com/article/10 .3390/nu14030520/s1, Table S1: Search terms for study screening, Table S2: The quality assessment of included studies.

Author Contributions: Conceptualization and methodology, T.S., D.T. and N.L.; software, D.T. and N.L.; validation, T.S., D.T., J.M. and N.L.; analysis, T.S. and D.T.; supervision, T.S. and N.L.; draft manuscript preparation, T.S. and D.T.; writing and editing, T.S., D.T., J.M. and N.L. All authors have read and agreed to the published version of the manuscript.

Funding: This research received no external funding.

Institutional Review Board Statement: Not applicable.

Conflicts of Interest: The authors declare no conflict of interest for this work.

\section{References}

1. Centers for Disease Control and Prevention. International Statistics. Available online: https://www.cdc.gov/hiv/statistics/ overview /index.html (accessed on 15 December 2021).

2. HIV.gov. Global Statistics. Available online: https://www.hiv.gov/hiv-basics/overview/data-and-trends/global-statistics (accessed on 15 December 2021).

3. World Health Organization. Consolidated Guidelines on HIV Prevention, Testing, Treatment, Service Delivery and Monitoring: Recommendations for a Public Health Approach; World Health Organization: Geneva, Switzerland, 2021.

4. European Food Safety Authority. Food Supplements. Available online: https://www.efsa.europa.eu/en/topics/topic/foodsupplements (accessed on 15 December 2021).

5. Castleman, T.; Seumo-Fosso, E.; Cogill, B. Food and Nutrition Implications of Antiretroviral Therapy in Resource Limited Settings; Food and Nutrition Technical Assistance Project, Academy for Educational Development: Washington, DC, USA, 2004.

6. Tonui, K.K.; Njogu, E.; Onyango, A.C. Dietary intake of HIV-seropositive clients attending Longisa County Hospital Comprehensive Care Clinic, Bomet County, Kenya. S. Afr. J. Clin. Nutr. 2020, 33, 53-57. [CrossRef]

7. Singhal, N.; Austin, J. A Clinical Review of Micronutrients in HIV Infection. J. Int. Assoc. Physicians AIDS Care 2002, 1, 63-75. [CrossRef] [PubMed]

8. Koethe, J.R.; Chi, B.H.; Megazzini, K.M.; Heimburger, D.C.; Stringer, J.S.A. Macronutrient supplementation for malnourished HIV-infected adults: A review of the evidence in resource-adequate and resource-constrained settings. Clin. Infect. Dis. 2009, 49, 787-798. [CrossRef] [PubMed]

9. Lorenc, A.; Robinson, N. A review of the use of complementary and alternative medicine and HIV: Issues for patient care. AIDS Patient Care STDs 2013, 27, 503-510. [CrossRef] [PubMed]

10. Schmidt, L.E.; Dalhoff, K. Food-drug interactions. Drugs 2002, 62, 1481-1502. [CrossRef] [PubMed]

11. Esteves, F.; Rueff, J.; Kranendonk, M. The central role of cytochrome P450 in xenobiotic metabolism-A brief review on a fascinating enzyme family. J. Xenobiotics 2021, 11, 7. [CrossRef]

12. Carver, P.L.; Fleisher, D.; Zhou, S.Y.; Kaul, D.; Kazanjian, P.; Li, C. Meal Composition effects on the oral bioavailability of indinavir in HIV-infected patients. Pharm. Res. 1999, 16, 718-724. [CrossRef]

13. Deng, J.; Zhu, X.; Chen, Z.; Fan, C.H.; Kwan, H.S.; Wong, C.H.; Shek, K.Y.; Zuo, Z.; Lam, T.N. A review of food-drug interactions on oral drug absorption. Drugs 2017, 77, 1833-1855. [CrossRef]

14. Moore, K.H.P.; Shaw, S.; Laurent, A.L.; Lloyd, P.; Duncan, B.; Morris, D.M.; O’Mara, M.J.; Pakes, G.E. Lamivudine/zidovudine as a combined formulation tablet: Bioequivalence compared with lamivudine and zidovudine administered concurrently and the effect of food on absorption. J. Clin. Pharmacol. 1999, 39, 593-605. [CrossRef]

15. Roberts, J.L.; Kiser, J.J.; Hindman, J.T.; Meditz, A.L. Virologic failure with a raltegravir-containing antiretroviral regimen and concomitant calcium administration. Pharmacother. J. Hum. Pharmacol. Drug Ther. 2011, 31, 1042. [CrossRef]

16. Page, M.J.; McKenzie, J.; Bossuyt, P.; Boutron, I.; Hoffmann, T.; Mulrow, C.; Shamseer, L.; Tetzlaff, J.; Akl, E.; Brennan, S.E.; et al. The PRISMA 2020 statement: An updated guideline for reporting systematic reviews. BMJ 2021, 372, n71. [CrossRef] [PubMed]

17. Koziolek, M.; Alcaro, S.; Augustijns, P.; Basit, A.W.; Grimm, M.; Hens, B.; Hoad, C.L.; Jedamzik, P.; Madla, C.M.; Maliepaard, M.; et al. The mechanisms of pharmacokinetic food-drug interactions-A perspective from the UNGAP group. Eur. J. Pharm. Sci. 2019, 134, 31-59. [CrossRef] [PubMed]

18. Ma, L.-L.; Wang, Y.-Y.; Yang, Z.-H.; Huang, D.; Weng, H.; Zeng, X.-T. Methodological quality (risk of bias) assessment tools for primary and secondary medical studies: What are they and which is better? Mil. Med. Res. 2020, 7, 7. [CrossRef] [PubMed] 
19. Sterne, J.A.C.; Hernán, M.A.; Reeves, B.C.; Savović, J.; Berkman, N.D.; Viswanathan, M.; Henry, D.; Altman, D.G.; Ansari, M.T.; Boutron, I.; et al. ROBINS-I: A tool for assessing risk of bias in non-randomised studies of interventions. BMJ 2016, 355 , i4919. [CrossRef]

20. Higgins, J.P.T.; Thompson, S.G.; Deeks, J.J.; Altman, D.G. Measuring inconsistency in meta-analyses. BMJ 2003, 327, 557-560. [CrossRef]

21. Herzog, R.; Álvarez-Pasquin, M.J.; Díaz, C.; del Barrio, J.L.; Estrada, J.M.; Gil, Á. Are healthcare workers' intentions to vaccinate related to their knowledge, beliefs and attitudes? A systematic review. BMC Public Health 2013, 13, 154. [CrossRef]

22. Murad, M.H.; Sultan, S.; Haffar, S.; Bazerbachi, F. Methodological quality and synthesis of case series and case reports. BMJ Evid. Based Med. 2018, 23, 60-63. [CrossRef]

23. Ding, H.; Hu, G.L.; Zheng, X.Y.; Chen, Q.; Threapleton, D.E.; Zhou, Z.H. The method quality of cross-over studies involved in Cochrane Systematic Reviews. PLoS ONE 2015, 10, e0120519. [CrossRef]

24. Kupferschmidt, H.H.T.; Fattinger, K.E.; Ha, H.R.; Follath, F.; Krähenbühl, S. Grapefruit juice enhances the bioavailability of the HIV protease inhibitor saquinavir in man. Br. J. Clin. Pharmacol. 1998, 45, 355-359. [CrossRef]

25. Yuen, G.J.; Lou, Y.; Thompson, N.F.; Otto, V.R.; Allsup, T.L.; Mahony, W.B.; Hutman, H.W. Abacavir/lamivudine/zidovudine as a combined formulation tablet: Bioequivalence compared with each component administered concurrently and the effect of food on absorption. J. Clin. Pharmacol. 2001, 41, 277-288. [CrossRef]

26. Penzak, S.R.; Acosta, E.P.; Turner, M.; Edwards, D.J.; Hon, Y.Y.; Desai, H.D.; Jann, M.W. Effect of Seville orange juice and grapefruit juice on indinavir pharmacokinetics. J. Clin. Pharmacol. 2002, 42, 1165-1170. [CrossRef] [PubMed]

27. Falcoz, C.; Jenkins, J.M.; Bye, C.; Hardman, T.C.; Kenney, K.B.; Studenberg, S.; Fuder, H.; Prince, W.T. Pharmacokinetics of GW433908, a prodrug of amprenavir, in healthy male volunteers. J. Clin. Pharmacol. 2002, 42, 887-898. [CrossRef] [PubMed]

28. Piscitelli, S.C.; Burstein, A.H.; Welden, N.; Gallicano, K.D.; Falloon, J. The effect of garlic supplements on the pharmacokinetics of saquinavir. Clin. Infect. Dis. 2002, 34, 234-238. [CrossRef] [PubMed]

29. Slain, D.; Amsden, J.R.; Khakoo, R.A.; Fisher, M.A.; Lalka, D.; Hobbs, G.R. Effect of high-dose vitamin C on the steady-state pharmacokinetics of the protease inhibitor indinavir in healthy volunteers. Pharmacotherapy 2005, 25, 165-170. [CrossRef]

30. Mouly, S.J.; Matheny, C.; Paine, M.F.; Smith, G.; Lamba, J.; Lamba, V.; Pusek, S.N.; Schuetz, E.G.; Stewart, P.W.; Watkins, P.B. Variation in oral clearance of saquinavir is predicted by CYP3A $5^{*} 1$ genotype but not by enterocyte content of cytochrome P450 3A5. Clin. Pharmacol. Ther. 2005, 78, 605-618. [CrossRef]

31. DiCenzo, R.; Frerichs, V.; Larppanichpoonphol, P.; Predko, L.; Chen, A.; Reichman, R.; Morris, M. Effect of quercetin on the plasma and intracellular concentrations of saquinavir in healthy adults. Pharmacother. J. Hum. Pharmacol. Drug Ther. 2006, 26, 1255-1261. [CrossRef]

32. Sekar, V.; Kestens, D.; Spinosa-Guzman, S.; de Pauw, M.; de Paepe, E.; Vangeneugden, T.; Lefebvre, E.; Hoetelmans, R.M.W. The effect of different meal types on the pharmacokinetics of darunavir (TMC114)/ritonavir in HIV-negative healthy volunteers. J. Clin. Pharmacol. 2007, 47, 479-484. [CrossRef]

33. Robertson, S.M.; Davey, R.T.; Voell, J.; Formentini, E.; Alfaro, R.M.; Penzak, S.R. Effect of Ginkgo biloba extract on lopinavir, midazolam and fexofenadine pharmacokinetics in healthy subjects. Curr. Med. Res. Opin. 2008, 24, 591-599. [CrossRef]

34. Patel, P.; Song, I.; Borland, J.; Patel, A.; Lou, Y.; Chen, S.; Wajima, T.; Peppercorn, A.; Min, S.S.; Piscitelli, S.C. Pharmacokinetics of the HIV integrase inhibitor S/GSK1349572 co-administered with acid-reducing agents and multivitamins in healthy volunteers. J. Antimicrob. Chemother. 2011, 66, 1567-1572. [CrossRef]

35. Calderón, M.M.; Chairez, C.L.; Gordon, L.A.; Alfaro, R.M.; Kovacs, J.A.; Penzak, S.R. Influence of Panax ginseng on the steady state pharmacokinetic profile of lopinavir-ritonavir in healthy volunteers. Pharmacother. J. Hum. Pharmacol. Drug Ther. 2014, 34, 1151-1158. [CrossRef]

36. Song, I.; Borland, J.; Arya, N.; Wynne, B.; Piscitelli, S. Pharmacokinetics of dolutegravir when administered with mineral supplements in healthy adult subjects. J. Clin. Pharmacol. 2015, 55, 490-496. [CrossRef] [PubMed]

37. Buchanan, A.M.; Holton, M.; Conn, I.; Davies, M.; Choukour, M.; Wynne, B.R. Relative bioavailability of a dolutegravir dispersible tablet and the effects of low- and high-mineral-content water on the tablet in healthy adults. Clin. Pharmacol. Drug Dev. 2017, 6, 577-583. [CrossRef] [PubMed]

38. Yamada, H.; Ikushima, I.; Nemoto, T.; Ishikawa, T.; Ninomiya, N.; Irie, S. Effects of a nutritional protein-rich drink on the pharmacokinetics of elvitegravir, cobicistat, emtricitabine, tenofovir alafenamide, and tenofovir compared with a standard meal in healthy Japanese male subjects. Clin. Pharmacol. Drug Dev. 2017, 7, 132-142. [CrossRef] [PubMed]

39. Yonemura, T.; Okada, N.; Sagane, K.; Okamiya, K.; Ozaki, H.; Iida, T.; Yamada, H.; Yagura, H. Effects of milk or apple juice ingestion on the pharmacokinetics of elvitegravir and cobicistat in healthy Japanese male volunteers: A randomized, single-dose, three-way crossover study. Clin. Pharmacol. Drug Dev. 2018, 7, 737-743. [CrossRef]

40. Jensen-Fangel, S.; Justesen, U.S.; Black, F.T.; Pedersen, C.; Obel, N. The use of calcium carbonate in nelfinavir-associated diarrhoea in HIV-1-infected patients. HIV Med. 2003, 4, 48-52. [CrossRef]

41. Sheehan, N.L.; van Heeswijk, R.P.G.; Foster, B.C.; Akhtar, H.; Singhal, N.; Seguin, I.; DelBalso, L.; Bourbeau, M.; Chauhan, B.M.; Boulassel, M.-R.; et al. The Effect of $\beta$-carotene supplementation on the pharmacokinetics of nelfinavir and its active metabolite M8 in HIV-1-infected patients. Molecules 2012, 17, 688-702. [CrossRef] 
42. Abdissa, A.; Olsen, M.F.; Yilma, D.; Tesfaye, M.; Girma, T.; Christiansen, M.; Hagen, C.; Wiesner, L.; Castel, S.; Aseffa, A.; et al. Lipid-based nutrient supplements do not affect efavirenz but lower plasma nevirapine concentrations in Ethiopian adult HIV patients. HIV Med. 2015, 16, 403-411. [CrossRef]

43. Munkombwe, D.; Muungo, T.L.; Michelo, C.; Kelly, P.; Chirwa, S.; Filteau, S. Lipid-based nutrient supplements containing vitamins and minerals attenuate renal electrolyte loss in HIV/AIDS patients starting antiretroviral therapy: A randomized controlled trial in Zambia. Clin. Nutr. ESPEN 2016, 13, e8-e14. [CrossRef]

44. Daskapan, A.; Dijkema, D.; de Weerd, D.A.; Bierman, W.F.W.; Kosterink, J.G.W.; van der Werf, T.S.; Alffenaar, J.-W.C.; Stienstra, Y. Food intake and darunavir plasma concentrations in people living with HIV in an outpatient setting. Br. J. Clin. Pharmacol. 2017, 83, 2325-2329. [CrossRef]

45. Cochrane Handbook for Systematic Reviews of Interventions. Recommendations on Testing for Funnel Plot Asymmetry. Available online: https://handbook-5-1.cochrane.org/chapter_10/10_4_3_1_recommendations_on_testing_for_funnel_plot_asymmetry. htm (accessed on 15 December 2021).

46. Barry, M.; Gibbons, S.; Back, D.; Mulcahy, F. Protease inhibitors in patients with HIV disease. Clin. Pharmacokinet. 1997, 32, 194-209. [CrossRef]

47. Kiani, J.; Imam, S.Z. Medicinal importance of grapefruit juice and its interaction with various drugs. Nutr. J. 2007, 6, 33. [CrossRef] [PubMed]

48. Boffito, M.; Acosta, E.; Burger, D.; Fletcher, C.V.; Flexner, C.; Garaffo, R.; Gatti, G.; Kurowski, M.; Perno, C.F.; Peytavin, G.; et al. Part 1 Current status and future prospects of therapeutic drug monitoring and applied clinical pharmacology in antiretroviral therapy. Antivir. Ther. 2005, 10, 375-392. [PubMed]

49. Usach, I.; Melis, V.; Peris, J.-E. Non-nucleoside reverse transcriptase inhibitors: A review on pharmacokinetics, pharmacodynamics, safety and tolerability. J. Int. AIDS Soc. 2013, 16, 18567. [CrossRef] [PubMed]

50. SUSTIVA $^{\circledR}$. (Efavirenz) Capsules and Tablets. Available online: https://www.accessdata.fda.gov/drugsatfda_docs/label/2008 /020972s030,021360s019lbl.pdf (accessed on 15 December 2021).

51. Holec, A.D.; Mandal, S.; Prathipati, P.K.; Destache, C.J. Nucleotide reverse transcriptase inhibitors: A thorough review, present status and future perspective as HIV therapeutics. Curr. HIV Res. 2017, 15, 411-421. [CrossRef]

52. Annex, I. Summary of Product Characteristics. Available online: https://www.ema.europa.eu/en/documents/productinformation/descovy-epar-product-information_en.pdf (accessed on 15 December 2021).

53. Highlights of Prescribing Information. Available online: https://www.accessdata.fda.gov/drugsatfda_docs/label/2013/20479 0lbl.pdf (accessed on 15 December 2021).

54. Starup-Linde, J.; Rosendahl, S.B.; Storgaard, M.; Langdahl, B. Management of osteoporosis in patients living with HIV-A systematic review and meta-analysis. JAIDS J. Acquir. Immune Defic. Syndr. 2020, 83, 1-8. [CrossRef]

55. Mori, T.; Itoh, S.; Ohgiya, S.; Ishizaki, K.; Kamataki, T. Regulation of CYP1A and CYP3A mRNAs by ascorbic acid in guinea pigs. Arch. Biochem. Biophys. 1997, 348, 268-277. [CrossRef]

56. Zannoni, V.G.; Flynn, E.J.; Lynch, M. Ascorbic acid and drug metabolism. Biochem. Pharmacol. 1972, 21, 1377-1392. [CrossRef]

57. Sato, P.H.; Zannoni, V.G. Stimulation of drug metabolism by ascorbic acid in weanling guinea pigs. Biochem. Pharmacol. 1974, 23, 3121-3128. [CrossRef]

58. Rikans, L.E.; Smith, C.R.; Zannoni, V.G. Ascorbic acid and cytochrome P-450. J. Pharmacol. Exp. Ther. 1978, 204, 702-713.

59. Netke, S.P.; Roomi, M.W.; Tsao, C.; Niedzwiecki, A. Ascorbic acid protects guinea pigs from acute aflatoxin toxicity. Toxicol. Appl. Pharmacol. 1997, 143, 429-435. [CrossRef]

60. van Heeswijk, R.P.G.; Cooper, C.L.; Foster, B.C.; Chauhan, B.M.; Shirazi, F.; Seguin, I.; Phillips, E.J.; Mills, E. Effect of high-dose vitamin C on hepatic cytochrome P450 3A4 activity. J. Hum. Pharmacol. Drug Ther. 2005, 25, 1725-1728. [CrossRef] [PubMed]

61. Foster, B.C.; Foster, M.S.; VandenHoek, S.; Krantis, A.; Budzinski, J.W.; Arnason, J.T.; Gallicano, K.D.; Choudri, S. An in vitro evaluation of human cytochrome P450 3A4 and P-glycoprotein inhibition by garlic. J. Pharm. Pharm. Sci. 2001, 4, 176-184. [PubMed]

62. Ho, B.E.; Shen, D.D.; McCune, J.S.; Bui, T.; Risler, L.; Yang, Z.; Ho, R.J.Y. Effects of garlic on cytochromes P450 2C9- and 3A4-mediated drug metabolism in human hepatocytes. Sci. Pharm. 2010, 78, 473-481. [CrossRef] [PubMed]

63. Jacek, H.; Rentsch, K.M.; Steinert, H.C.; Pauli-Magnus, C.; Meier, P.J.; Fattinger, K. No effect of garlic extract on saquinavir kinetics and hepatic CYP3A4 function measured by the erythromycin breath test. Clin. Pharmacol. Ther. 2004, 75, P80. [CrossRef]

64. Obach, R.S. Inhibition of human cytochrome P450 enzymes by constituents of St. John's Wort, an herbal preparation used in the treatment of depression. J. Pharmacol. Exp. Ther. 2000, 294, 88-95. [PubMed]

65. Limtrakul, P.; Khantamat, O.; Pintha, K. Inhibition of P-glycoprotein function and expression by kaempferol and quercetin J. Chemother. 2005, 17, 86-95. [CrossRef]

66. Shinozuka, K.; Umegaki, K.; Kubota, Y.; Tanaka, N.; Mizuno, H.; Yamauchi, J.; Nakamura, K.; Kunitomo, M. Feeding of Ginkgo biloba extract (GBE) enhances gene expression of hepatic cytochrome P-450 and attenuates the hypotensive effect of nicardipine in rats. Life Sci. 2002, 70, 2783-2792. [CrossRef]

67. Kubota, Y.; Kobayashi, K.; Tanaka, N.; Nakamura, K.; Kunitomo, M.; Umegaki, K.; Shinozuka, K. Interaction of Ginkgo biloba extract (GBE) with hypotensive agent, nicardipine, in rats. In Vivo 2003, 17, 409-412. 
68. Malati, C.Y.; Robertson, S.M.; Hunt, J.D.; Chairez, C.; Alfaro, R.M.; Kovacs, J.A.; Penzak, S.R. Influence of Panax ginseng on cytochrome P450 (CYP)3A and P-glycoprotein (P-gp) activity in healthy participants. J. Clin. Pharmacol. 2012, 52, 932-939. [CrossRef]

69. Foster, B.; Francovic, A.; Drouin, C.; Akhtar, H.; Cameron, W. Trans-b-carotene and retinoids can effect human cytochrome p450 3a4-mediated metabolism (Abstract tupeb4564). In Proceedings of the XIV International AIDS Conference, Barcelona, Spain, 7-12 July 2002.

70. Sczech, R.; Landes, N.; Pfluger, P.; Kluth, D.; Schweigert, F.J. Carotenoids and their metabolites are naturally occurring activators of gene expression via the pregnane $X$ receptor. Eur. J. Nutr. 2004, 43, 336-343.

71. Higgins, J.P.; Thomas, J.; Chandler, J.; Cumpston, M.; Li, T.; Page, M.J.; Welch, V.A. Cochrane Handbook for Systematic Reviews of Interventions; John Wiley \& Sons: Chichester, UK, 2019. 